\title{
ENHANCING STUDENTS' SPATIAL REASONING SKILLS WITH ROBOTICS INTERVENTION
}

\author{
Krista Francis \\ University of Calgary \\ kfrancis@ucalgary.ca \\ Dana Poscente \\ University of Calgary \\ djposcen@ucalgary.ca
}

\author{
Stefan Rothschuh \\ University of Calgary \\ stefan.rothschuh@ucalgary.ca \\ Brent Davis \\ University of Calgary \\ abdavis@ucalgary.ca
}

Spatial reasoning is a high-impact topic as it strongly predicts interest in, appreciation of, and success in STEM domains and careers. Yet, spatial reasoning is often under-used, underdeveloped, and ignored in current grade-school curriculum and teaching. Framed by the perspective of embodied cognition, our study explores changes in elementary students' spatial reasoning skills after participation in either a short-term or a long-term robotics intervention. We administered measures of spatial reasoning elements before and after two differently structured robotics interventions to students aged 9-10 years: a short-term $(N=11)$ and two long-term $(N=48)$. Statistical analysis revealed significant improvements to several different elements of spatial reasoning in both groups. Our findings suggest that programming robots in either the short- or long-term leads to improvements in spatial reasoning.

Keywords: Programming and coding; STEM / STEAM; Technology

The purpose of our study is to report changes in elementary students' spatial reasoning skills after participation in either a short-term or a long-term robotics intervention. Spatial reasoning is a highimpact topic as it strongly predicts interest in, appreciation of, and success in STEM domains and careers (Casey et al., 2011; Lubinski, 2010; Mix \& Cheng, 2012; Mix et al., 2016; Wai et al., 2009). Spatial reasoning is highly malleable, and can be learned (Julià \& Antolí, 2016; Sorby, 2009; Uttal et al., 2013).Yet, spatial reasoning is mostly under-used, underdeveloped, and ignored in current gradeschool curriculum and teaching (Newcombe, 2010). A few studies have investigated the positive effects of spatial reasoning and robotics interventions in schools (Coxon, 2012; Francis et al., 2016; Julià \& Anatolì, 2016, Julià \& Anatolì, 2018; Khan et al., 2014; Verner, 2004). Our current study builds on previous studies to further investigate the malleability of spatial reasoning with short-term and long-term robotics interventions.

\section{What is spatial reasoning?}

\section{Background Literature}

While there is considerable debate about what spatial reasoning is (see Uttal et al., 2013), we draw upon Bruce et al.'s (2017) definition of spatial reasoning as the ability to recognize and (mentally) manipulate the spatial properties of objects and the spatial relations among objects. Davis et al. (2015, p. 141) describe the emergent complexity of spatial reasoning skills as co-evolved and complementary nature of the mental and physical actions. The nature of these skills is entangled and emergent. Their description of spatial reasoning elements include ALTERING (dilating/contracting, distorting/morphing, scaling, folding, shearing), MOVING (sliding, rotating, reflecting, balancing, sliding), SITUATING (dimension shifting, locating, orienting, pathfinding, intersecting), SENSATING (perspective-taking, visualizing, propriocepting, imagining, tactilizing), INTERPRETING (diagramming, modeling, symmetrizing, comparing, relating), [DE]CONSTRUCTING (de/re/composing, un/re/packing, re/arranging, sectioning, fitting). 


\section{Context}

This study is based on a multi-year design-based research project investigating how robotics influences spatial reasoning. The data reported here is based on quasi experimental results investigating if robotics interventions impact spatial reasoning. The first iteration of the design reported here was based on a week-long robotics academy with elementary teachers and Grade 4-5 students held at a university (15 hours). The second and third iterations of the design reported here were year-long classroom interventions at a local school (40 hours) in Grades 4-5. It is beyond the confines of this brief research report to describe the structures of the interventions in detail. However, Table 1 summarizes the sequence and spatial reasoning elements engaged in each task. Every robotics task of the short-term and the long-term intervention involved multiple spatial elements.

Table 1. Spatial Skills Found in the Intervention Task

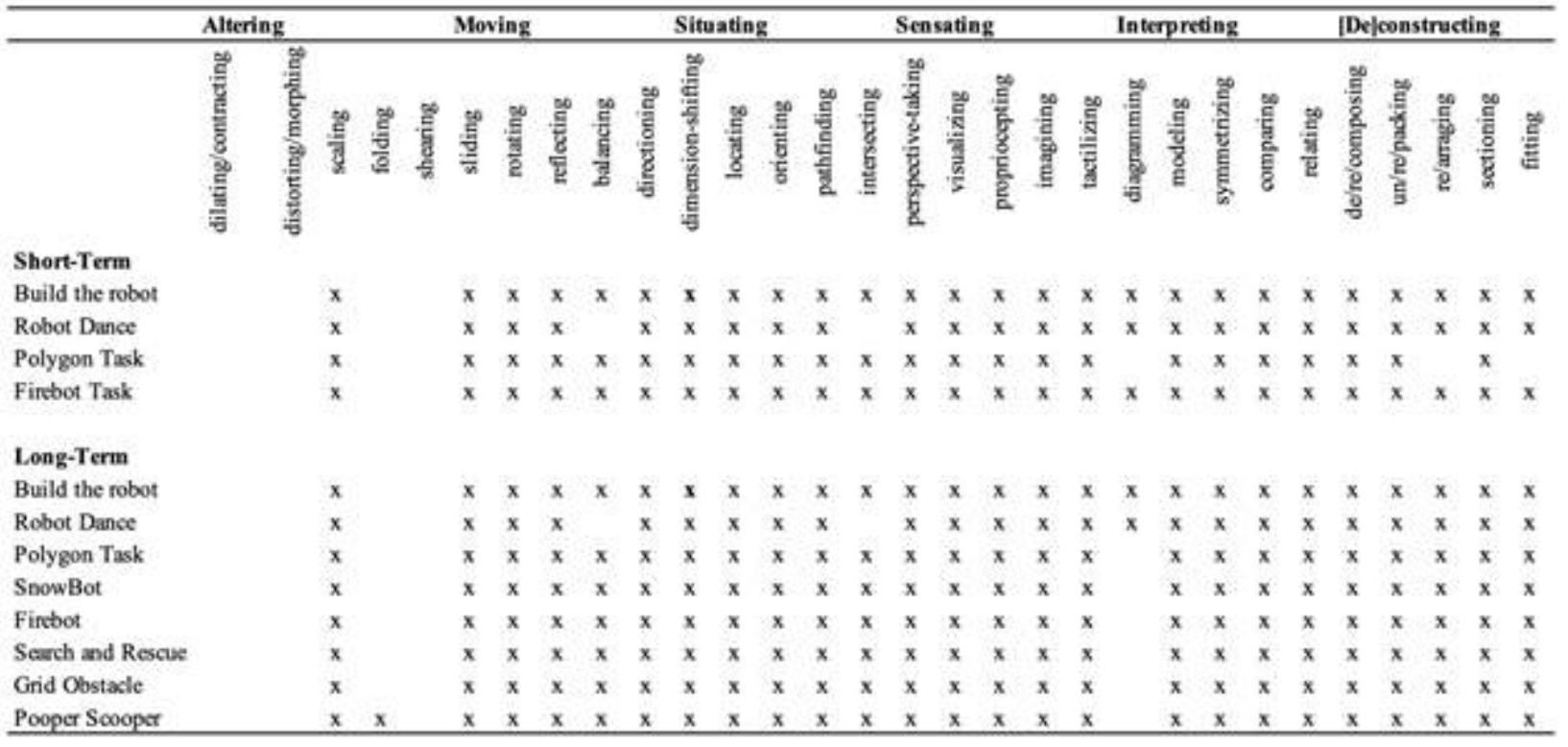

\section{Methodology}

Our study explores the following research question: Do elementary students' spatial reasoning skills improve after participation in either a short-term or a long-term robotics intervention? The data reported here is based on the quasi experimental pre- and post-test results collected before and after the interventions mentioned above. Participants in the short-term intervention included 11 Grade 4 students. Participants in the long-term intervention were from a different school and included 48 students total, whereby ten Grade 4 students completed the pre- and post-tests in 2017-2018. During the following school year 2018-2019, 19 Grade 4 students and 19 Grade 5 students respectively took the pre- and post-tests on spatial reasoning skills. The administration of the pre- and post-test instruments was similar for the short- and long-term interventions.

Description of pre- and post-test instrument. We developed an instrument that encompassed a broad range of spatial skills based on an amalgamation of established protocols. The spatial reasoning pre- post-test consisted of seven (different) task categories with 13 test items for the shortterm intervention, and three additional test items for the long-term intervention. The short-term preand post-test included four Sorby drawing items, two paper folding items, two shape rotation items, two stereonet items, one building a 3D object from an image item, one moving a shape on a grid item, and one cross-section of a cube: isometric projection. The three additional items in the reasoning pre- and post-test of the long-term intervention included one more paper folding item, a 
block visualization/rotation item, and a pattern arranging item. Groups of three students were tested at a time. Each of the tests took approximately 45 minutes.

Analysis of results of short-term intervention. The data reported in this section details the Grade 4 results from the week-long intervention in 2016. The data consisted of one group of the same subjects at two different points in time (before and after the intervention). For such data, the Paired tTest is an appropriate test to compare the means when the data is normally distributed (Huck, 2012; Mills \& Gay, 2019). For data that is not normally distributed, the non-parametric Wilcoxon SignedRanks Test is more appropriate (Huck, 2012; Mills \& Gay, 2019). To determine if that data is normally distributed, the Shapiro Wilks test is a suitable parametric test for a small sample size (Huck, 2012; Mills \& Gay, 2019).

To compare spatial reasoning at the beginning and end of the week long robotics camp (15 hours) a paired t-test was conducted on the normally distributed Items 2, 3, 5, 10, 11, 12, and Total (pre- and post-test) and a Wilcoxon signed-rank test was used for the not normally distributed Items 1, 4, 6, 7, 8, 9, and 13 (pre- and post-test). All spatial reasoning test items saw improvement. There was a significant improvement for the Sorby drawing Item 2: $t(10)=-2.4, \mathrm{p}<.05$; building a $3 \mathrm{D}$ object from a picture Item 11: $t(36)=-2.9, \mathrm{p}=.05$, moving a shape on a grid Item 12: $t(11)=-2.4, \mathrm{p}=0.05$ and; the overall total: $t(11)=-3.05, \mathrm{p}<.05$.

Analysis of Results of Year Long Intervention. To compare spatial reasoning at the beginning and end of the school year a paired t-test was conducted on normally distributed Items 1-16 (pre- and post-test) and a Wilcoxon signed-rank test was used on the non-normally distributed Items Total (pre- and post-test). The data combines the Grade 4 results from Year 2017-2018 and the Grade 4 and 5 results from Year 2018-2019. All spatial reasoning test items saw improvement. There was a significant improvement for the Sorby drawing Item 2: $\mathrm{t}(47)=-2.4, \mathrm{p}<.05$; paper folding Item 5: $\mathrm{t}(47)=-3.4, \mathrm{p}<.05$; rotation Item $9: \mathrm{t}(47)=-3.0, \mathrm{p}<.05$; pattern arranging Item $16: \mathrm{t}(36)=-4.2, \mathrm{p}<$ .05 , and; the overall total: $\mathrm{Z}(-4.519), \mathrm{p}<.05$.

\section{Discussion}

In this study, we found how powerfully robotics interventions can improve spatial reasoning. We found similar results from both the short-term and long-term interventions. For the short-term significant results were observed in Sorby drawing, building a 3D object from a picture, moving a shape on a grid, and overall total score. For the long-term, significant results were observed in Sorby drawing, paper folding, shape rotation, pattern arranging and overall total score. The long-term intervention did not reveal a significant improvement to building 3D object from picture and moving along a grid. Perhaps this is because the students in the long-term study were already quite high in these two measures compared to the short-term group; there was not as much room to improve with these groups.

Previous studies also found improvements in spatial reasoning with robotics interventions. Verner (2004) reported improvements in three spatial reasoning skills: visualization, perception, rotation. Julià and Anatolì $(2016 ; 2018)$ found improvements with spatial reasoning tasks. By drawing upon Davis et al.'s (2015) descriptions of spatial reasoning elements, we were able to provide a broader perspective of spatial reasoning than Verner (2004). Like Julià and Anatolì (2016; 2018), our instrument included paper folding, shape rotation, cube comparison and perspective taking. However, our test instrument also included Sorby drawing items, stereonets, building 3D objects from pictures, moving shapes on a grid and isometric projects. We not only looked at the spatial reasoning tasks as did Julià and Anatolì $(2016$; 2018), we also identified the spatial reasoning elements that were associated with each task. For example, visualization and imagining from SENSTATING were elements that appeared in every task and are integral to spatial reasoning as evident from most definitions (see Uttal et al., 2013). 
Our identification of spatial elements within the tasks was important to show the compatibility of the test instruments with robotics. For instance, it may seem like the Sorby drawing item is completely different from programming a robot to move. However, the Sorby drawing item requires seven spatial reasoning elements: SITUATING (dimension shifting, intersecting), SENSATING (visualizing, imagining), INTERPRETING (diagramming), and [DE]CONSTRUCTING (de/re/composing, fitting). Francis et al. (2016) observed the engagement of these same spatial reasoning elements when students programmed robots to move.

Our findings complement our previous qualitative research which illustrated how spatial reasoning is engaged while building (Khan et al., 2014) and programming robots to move (Francis et al., 2016). These prior studies illustrated the complex and co-emergent nature of spatial reasoning which helped provide a basis for this current study that drew upon embodied cognition to investigate the results of learning from action. Our results provide some validation to Pouw et al.'s (2014) prediction that sensorimotor experiences are important for development of related concepts. In other words, this study along with our two previous studies (Khan et al., 2015; Francis et al., 2016) illustrate the power of sensorimotor experiences of robotics learning for improving spatial reasoning. Not only is spatial reasoning engaged, it also improves significantly.

As future work, we would like to explore how programming robots to move helps with learning mathematics. Spatial reasoning ability is correlated to mathematics achievement (Mix \& Cheng, 2012) and computer programming is highly related to mathematics. Exploring connections could lead to unexpected and emerging insights for the teaching and learning of mathematics.

\section{References}

Bruce, C. D., Davis, B., Sinclair, N., McGarvey, L., Hallowell, D., Drefs, M., ... Woolcott, G. (2017). Understanding gaps in research networks: using "spatial reasoning" as a window into the importance of networked educational research. Educational Studies in Mathematics, 95(2), 143-161. https://doi.org/10.1007/s10649-016-9743-2.

Casey, B. M., Dearing, E., Vasilyeva, M., Ganley, C. M., \& Tine, M. (2011). Spatial and Numerical Predictors of Measurement Performance: The Moderating Effects of Community Income and Gender. Journal of Educational Psychology, 103(2), 296-311.

Cator, K. (2019). Digital Promise Accelerating Innovation in Education [Blog post]. Retrieved from https://digitalpromise.org/2019/01/09/closing-the-digital-learning-gap/.

Coxon, S. V. (2012). The malleability of spatial ability under treatment of a First LEGO League-based robotics simulation. Journal for the Education of the Gifted, 35(3), 291-316. https://doi.org/10.1177/0162353212451788

Davis, B., Okamoto, Y., \& Whiteley, W. (2015). Spatializing school mathematics. In Spatial Reasoning in the Early Years: Principles, Assertions, and Speculations (pp. 139-150). Routledge.

Francis, K., Khan, S., \& Davis, B. (2016). Enactivism, spatial reasoning and coding. Digital Experiences in Mathematics Education, 2(1), 1-20. https://doi.org/10.1007/s40751-015-0010-4

Huck, S. W. (2012). Reading statistics and research (6th ed.). Pearson.

Julià, C., \& Antolí, J. Ò. (2016). Spatial ability learning through educational robotics. International Journal of Technology and Design Education, 26(2), 185-203. https://doi.org/10.1007/s10798-015-9307-2

Julià, C., \& Antolì, J. Ò. (2018). Enhancing spatial ability and mechanical reasoning through a STEM course. International Journal of Technology and Design Education, 28(4), 957-983. https://doi.org/10.1007/s10798017-9428-x

Khan, S., Francis, K., \& Davis, B. (2015). Accumulation of experience in a vast number of cases: enactivism as a fit framework for the study of spatial reasoning in mathematics education. ZDM Mathematics Education, 47(2), 269-279. https://doi.org/10.1007/s11858-014-0623-x

Lubinski, D. (2010). Spatial ability and STEM: A sleeping giant for talent identification and development. Personality and Individual Differences, 49(4), 344-351. https://doi.org/10.1016/j.paid.2010.03.022

Mills, G., \& Gay, L.R. (2019). Educational research: Competencies for analysis and applications (12 ${ }^{\text {th }}$ ed.). Pearson. 
Mix, K. S., \& Cheng, Y.-L. (2012). The relation between space and math: developmental and educational implications. Advances in Child Development and Behavior, 42, 197-243. Retrieved from http://www.ncbi.nlm.nih.gov/pubmed/22675907.

Mix, K. S., Levine, S. C., Cheng, Y.-L., Young, C., Hambrick, D. Z., Ping, R., \& Konstantopoulos, S. (2016). Separate but correlated: the latent structure of space and mathematics across development. Journal of Experimental Psychology: General, 145(9), 1206-1227. https://doi.org/10.1037/xge0000182

Newcombe, N. S. (2010). Picture this: Increasing math and science learning by improving spatial thinking. American Educator, 34(2), 29-35.

Pouw, W. T. J. L., Gog, T. van, \& Paas, F. (2014). An embedded and embodied cognition review of instructional manipulatives. Educational Psychology Review, 26(1), 51-72. https://doi.org/10.1007/s10648-014-9255-5

Sorby, S. A. (2009). Educational Research in Developing 3-D Spatial Skills for Engineering Students. International Journal of Science Education, 31(3), 459-480.

Uttal, D. H., Meadow, N. G., Tipton, E., Hand, L. L., Alden, A. R., Warren, C., \& Newcombe, N. S. (2013). The malleability of spatial skills: A meta-analysis of training studies. Psychological Bulletin, 139(2), 352-402. https://doi.org/10.1037/a0028446

Verner, I. M. (2004). Robot manipulations: A synergy of visualization, computation and action for spatial instruction. International Journal of Computers for Mathematical Learning, 9(2), 213-234. https://doi.org/10.1023/B:IJCO.0000040892.46198.aa

Wai, J., Lubinski, D., \& Benbow, C. P. (2009). Spatial ability for STEM domains: Aligning over 50 years of cumulative psychological knowledge solidifies its importance. Journal of Educational Psychology, 101(4), 817835. https://doi.org/10.1037/a0016127workers/ 